\title{
Improved Secure Uncertain Robot by Artificial Based Adaptive Methodology
}

\author{
Farzin Piltan, Zahra Hivand, Sara Emamzadeh, Mina Mirzaie and Mohammad \\ Hossain Yarmahmoudi \\ Intelligent System and Robotic Lab, Iranian Institute of Advance Science and Technology \\ (IRAN SSP), Shiraz/Iran \\ piltan_f@iranssp.com,www.IRANSSP.COM/english
}

\begin{abstract}
This paper examines secure uncertain robot which performance is improved secure by artificial intelligence based on-line tuning method. Computed like torque (CLT) methodology is selected as a framework to construct the control law and address the better performance and reduce the error in presence of uncertainty in any trajectory. The main goal in security in any industrial factory is to guarantee acceptable trajectories tracking between the robot arm actual output and the desired input in presence of uncertainty and external disturbance. The proposed approach effectively combines the design technique from computed torque methodology is based on nonlinear stable system and fuzzy estimator to estimate the nonlinearity of undefined system dynamic in uncertain robot. The input represents the function between error and the rate of error. The outputs represent actual trajectory to improve the security, respectively. The fuzzy partly sliding switching methodology is on-line tune the computed torque like method based on adaptive methodology. The performance of the computed torque like method which controller coefficient is on-line tuned by fuzzy partly sliding switching algorithm (ACTLM) is validated the security through comparison with computed torque like methodology (CTLM). Simulation results signify good performance of trajectory in presence of uncertainty and external disturbance; it is used to show guarantee the security.
\end{abstract}

Keywords: uncertain robot, computed torque methodology, artificial intelligence, on-line tune, secure improved, sliding switching algorithm

\section{Introduction}

Robot manipulator is collection of links that connect by joints, these joints can be revolute and prismatic that revolute joint has rotary motion around an axis and prismatic joint has linear motion around an axis. Each joint provides one or more degrees of freedom (DOF). One of the most important challenges in the field of robot manipulator is control of it; because this system is multi input multi output (MIMO), nonlinear, time variant parameter and some dynamic parameters are uncertainty [1-2]. Presently, robot arms are used in different (unknown and/or unstructured) situation consequently caused to provide complicated systems, as a result strong mathematical theory are used in new control methodologies to design nonlinear robust methodology to guarantee the security in industrial factory. Classical and non-classical methods are two main categories of nonlinear plant control, where the conventional (classical) control theory uses the classical method and the non-classical control theory (e.g., fuzzy logic, neural network, and neuro fuzzy) uses the artificial intelligence methods. However both of conventional and artificial intelligence 
theories have applied effectively in many areas, but these methods also have some limitations to have an acceptable secure performance in unknown environment [3-4]. Modeling of an entire robot arm is a very important and complicated process because robot arms are nonlinear, MIMO and time variant. One purpose of accurate modeling is to save development costs of real robot arms and minimizing the risks of dangerous environment when validating controller designs [5].

Controller (control system) is a device which can sense information from linear or nonlinear system (e.g., robot arm) to improve the systems performance and the immune system behavior [3-20]. In feedback control system considering that there are many disturbances and also variable dynamic parameters something that is really necessary is keeping plant variables close to the desired value. Feedback control system development is the most important thing in many different fields of safety engineering. The main targets in designing control systems are safety stability, good disturbance rejection to reach the best safety, and small tracking error[6]. At present, in some applications robot arms are used in unknown and unstructured environment, therefore strong mathematical tools used in new control methodologies to design nonlinear robust controller with an acceptable safety performance (e.g., minimum error, good trajectory, disturbance rejection). One of the important nonlinear safety controllers is computed torque methodology which is used in nonlinear certain systems. One of the nonlinear stable controllers is computed torque controller, although this controller has been analyzed about industrial safety by many researchers but the first proposed was in the 1962 [7]. This methodology is used in wide range areas such as in safety control access process, in aerospace applications and in IC engines because this methodology can solve some main challenging topics in safety control access such as resistivity to the external disturbance and stability. Even though, this methodology is used in wide range areas but, pure computed torque method has an important drawbacks beside uncertain system and also in presence of external disturbance. Uncertainty in system can causes some problems about safety in industrial factory. To solve this problem, neural network, fuzzy logic, and neuro-fuzzy are synergically combined with nonlinear classical controller and used in nonlinear, time variant, and uncertainty plant (e.g., robot). Fuzzy logic methodology (FLM) is one of the most important applications of fuzzy logic theory [8]. This method can be used to resolve the challenge in nonlinear, uncertain, and noisy systems. This method is free of some model-based techniques as in classical controllers. Control robot arms using classical controllers are based on robot arm dynamic modelling. These controllers often have many problems for modelling. Conventional controllers require accurate information of dynamic model of robot arms. When the system model is unknown or when it is known but complicated, it is difficult or impossible to use classical mathematics to process this model[9]. The main reasons to use fuzzy logic technology are able to give approximate recommended solution for unclear and complicated systems to easy understanding and flexible. Fuzzy logic provides a method which is able to model a controller for nonlinear plant with a set of IFTHEN rules, or it can identify the control actions and describe them by using fuzzy rules [10]. In various dynamic parameters systems to have safety training, training on-line adaptive control methodology is used. Fuzzy partly sliding switching adaptive method is used in systems which want to training parameters by expert knowledge. This method guarantees self tuning stability in safety in nonlinear and uncertain robot. Mohan and Bhanot have addressed comparative study between some adaptive fuzzy, and a new hybrid fuzzy control algorithm for robot arm control. They found that self-organizing fuzzy logic controller and proposed hybrid integrator fuzzy give the best performance as well as simple structure. H.Temeltas has proposed fuzzy adaption techniques for VSC to achieve robust tracking of nonlinear systems and solves the chattering problem. Conversely system's performance is better than variable 
structure controller; it is depended on nonlinear dynamic equation. C. L. Hwang has proposed a Tagaki-Sugeno (TS) fuzzy model based variable structure control based on $N$ fuzzy based linear state-space to estimate the uncertainties. A MIMO FVSC reduces the chattering phenomenon and reconstructs the approximate the unknown system has been presented for a nonlinear system. Yoo and Ham have proposed a MIMO fuzzy system to help the compensation and estimation the torque coupling. This method can only tune the consequence part of the fuzzy rules. Medhafer has proposed an indirect adaptive fuzzy variable structure controller to control nonlinear system. This MIMO algorithm, applies to estimate the nonlinear dynamic parameters. Compared with the previous algorithm the numbers of fuzzy rules have reduced by introducing the variable structure surface as inputs of fuzzy systems. Y. Guo and P. Y. Woo have proposed a SISO fuzzy system compensate and reduce the chattering. C. M. Lin and C. F. Hsu can tune both systems by fuzzy rules. Shahnazi et al., have proposed a SISO PI direct adaptive fuzzy variable structure controller based on Lin and Hsu algorithm to reduce or eliminate chattering. The bounds of PI controller and the parameters are online adjusted by low adaption computation [11-16].

Problem statements: Even though, computed torque like methodology is used in wide range safety areas but, it also has limitation in presence of external disturbance [11]. To reduce the effect of external disturbance in proposed method, SISO novel fuzzy partly sliding switching adaptive method is applied to computed torque like method in robot arm.

Objectives: The main goal in this paper is to design a SISO fuzzy partly sliding switching adaptive computed torque like method which applied to robot arm. This method is easy to design and implement to have acceptable safety repose. Robot arm has nonlinear dynamic and uncertain parameters consequently; following objectives have been pursuit in the mentioned research: To develop a safety trajectory result in a position computed torque like method against uncertainties and to develop a position fuzzy partly sliding switching adaptive computed torque like methodology in order to solve the disturbance rejection.

This paper is organized as follows; section 2, is served as an introduction to the dynamic formulation of robot manipulator, introduction to nonlinear robust computed torque controller and fuzzy inference engine and its application to control and estimate dynamic uncertainty of robot manipulator. Part 3, introduces and describes the methodology. Section 4 presents the simulation results and discussion of this algorithm applied to a robot manipulator and the final section is describing the conclusion.

\section{Theory}

\section{A. Dynamic of robot arm:}

The equation of an $n-D O F$ robot manipulator governed by the following equation:

$$
M(q) \ddot{q}+N(q, \dot{q})=\tau
$$

Where $\tau$ is actuation torque, $\mathrm{M}(\mathrm{q})$ is a symmetric and positive define inertia matrix, $N(q, \dot{q})$ is the vector of nonlinearity term. This robot manipulator dynamic equation can also be written in a following form: 


$$
\tau=M(q) \ddot{q}+B(q)[\dot{q} \dot{q}]+C(q)[\dot{q}]^{2}+G(q)
$$

Where $\mathrm{B}(\mathrm{q})$ is the matrix of coriolios torques, $\mathrm{C}(\mathrm{q})$ is the matrix of centrifugal torques, and G(q) is the vector of gravity force. The dynamic terms in equation (2) are only manipulator position. This is a decoupled system with simple second order linear differential dynamics. In other words, the component $\ddot{q}$ influences, with a double integrator relationship, only the joint variable $q_{i}$, independently of the motion of the other joints. Therefore, the angular acceleration is found as to be:

$$
\ddot{\boldsymbol{q}}=\boldsymbol{M}^{-1}(q) .\{\tau-\boldsymbol{N}(\boldsymbol{q}, \dot{\boldsymbol{q}})\}
$$

This technique is very attractive from a control point of view.

\section{B. Computed torque methodology:}

Computed torque controller (CTC) is a powerful nonlinear controller which it widely used in control of robot manipulator. It is based on feedback linearization and computes the required arm torques using the nonlinear feedback control law. This controller works very well when all dynamic and physical parameters are known but when the robot manipulator has variation in dynamic parameters, in this situation the controller has no acceptable trajectory performance[14]. In practice, most of physical systems (e.g., robot manipulators) parameters are unknown or time variant, therefore, computed torque like controller used to compensate dynamic equation of robot manipulator. Research on computed torque controller is significantly growing on robot manipulator application. Vivas and Mosquera have proposed a predictive functional controller and compare to computed torque controller for tracking response in uncertain environment. However both controllers have been used in feedback linearization, but predictive strategy gives better result as a performance. A computed torque control with non parametric regression models have been presented for a robot arm. This controller also has been problem in uncertain dynamic models. Computed torque controller is a significant nonlinear controller to certain systems which it is based on feedback linearization and computes the required arm torques using the nonlinear feedback control law. When all dynamic and physical parameters are known, computed torque controller works fantastically; practically a large amount of systems have uncertainties, therefore computed torque like controller is the best case to solve this challenge[16].

The central idea of Computed torque methodology (CTM) is feedback linearization so, originally this algorithm is called feedback linearization controller. It has assumed that the desired motion trajectory for the manipulator $\boldsymbol{q}_{\boldsymbol{d}}(\boldsymbol{t})$, as determined, by a path planner. Defines the tracking error as:

$$
e(t)=q_{d}(t)-q_{a}(t)
$$

Where e(t) is error of the plant, $\boldsymbol{q}_{\boldsymbol{d}}(\boldsymbol{t})$ is desired input variable, that in our system is desired displacement, $\boldsymbol{q}_{\boldsymbol{a}}(\boldsymbol{t})$ is actual displacement. If an alternative linear state-space equation in the form $\dot{\boldsymbol{x}}=\boldsymbol{A} \boldsymbol{x}+\boldsymbol{B} \boldsymbol{U}$ can be defined as

$$
\dot{x}=\left[\begin{array}{ll}
\mathbf{0} & \boldsymbol{I} \\
\mathbf{0} & \mathbf{0}
\end{array}\right] \boldsymbol{x}+\left[\begin{array}{l}
\mathbf{0} \\
\boldsymbol{I}
\end{array}\right] \boldsymbol{U}
$$


With $\boldsymbol{U}=-\boldsymbol{M}^{-\mathbf{1}}(\boldsymbol{q}) \cdot \boldsymbol{N}(\boldsymbol{q}, \dot{\boldsymbol{q}})+\boldsymbol{M}^{-\mathbf{1}}(\boldsymbol{q}) \cdot \boldsymbol{\tau}$ and this is known as the Brunousky canonical form. By equation (4) and (5) the Brunousky canonical form can be written in terms of the state $\boldsymbol{x}=\left[\boldsymbol{e}^{T} \dot{\boldsymbol{e}}^{T}\right]^{T}$ as [1]:

$$
\frac{d}{d t}\left[\begin{array}{l}
e \\
\dot{e}
\end{array}\right]=\left[\begin{array}{ll}
0 & I \\
0 & 0
\end{array}\right] \cdot\left[\begin{array}{l}
e \\
\dot{e}
\end{array}\right]+\left[\begin{array}{l}
0 \\
I
\end{array}\right] U
$$

With

$$
U=\ddot{q}_{d}+M^{-1}(q) \cdot\{N(q \cdot \dot{q})-\tau\}
$$

Then compute the required arm torques using inverse of equation (7), is;

$$
\tau=M(q)(\ddot{q} d-U)+N(\dot{q}, q)
$$

This is a nonlinear feedback control law that guarantees tracking of desired trajectory. Selecting proportional-plus-derivative (PD) feedback for $\mathrm{U}(\mathrm{t})$ results in the PD-computed torque controller [6];

$$
\tau=M(q)\left(\ddot{q}_{d}+K_{v} \dot{e}+K_{p} e\right)+N(q, \dot{q})
$$

and the resulting linear error dynamics are

$$
\left(\ddot{q}_{d}+K_{v} \dot{e}+K_{p} e\right)=0
$$

According to the linear system theory, convergence of the tracking error to zero is guaranteed [6]. Where $\boldsymbol{K}_{\boldsymbol{p}}$ and $\boldsymbol{K}_{\boldsymbol{v}}$ are the controller gains. The result scheme shows in Figure 1 , in which two feedback loops, namely, inner loop and outer loop, which an inner loop is a compensate loop and an outer loop is a tracking error loop.

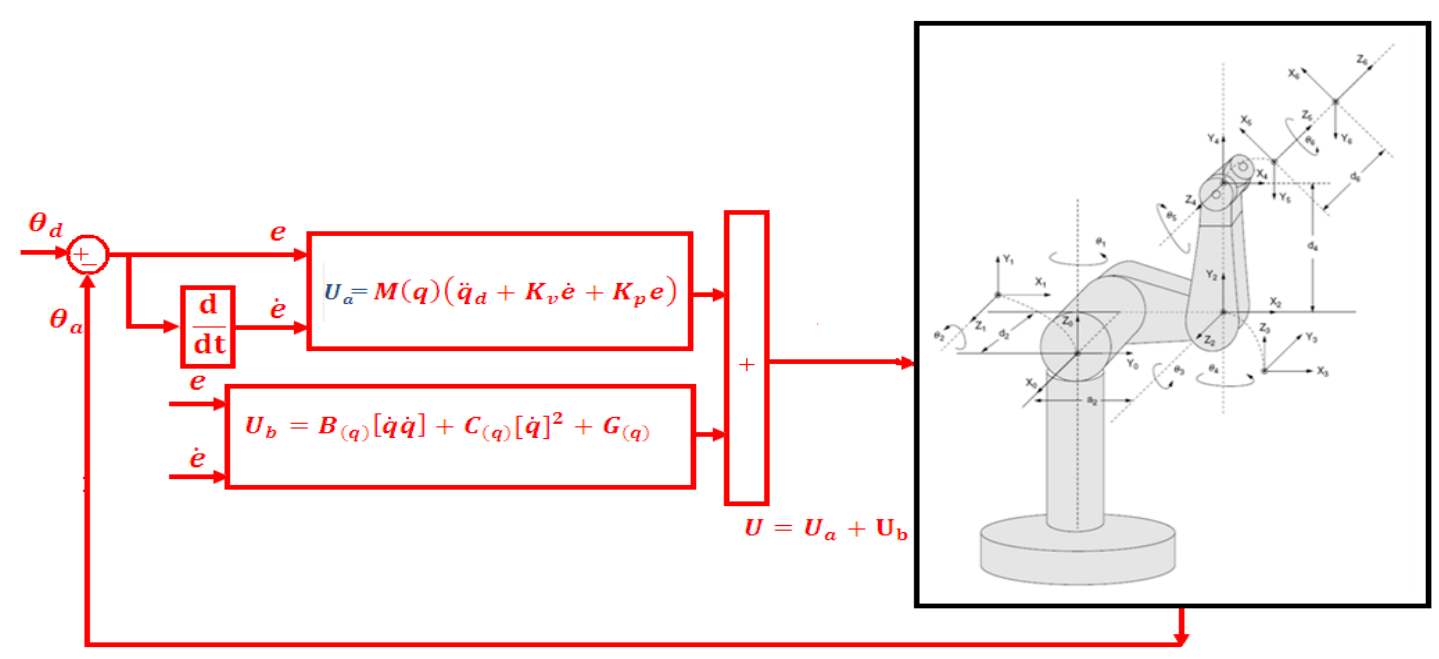

Figure 1. Block Diagram of PD-computed Torque Controller (PD-CTC) 
The application of proportional-plus-derivative (PD) computed torque controller to control of PUMA 560 robot manipulator introduced in this part. Suppose that in (9) the nonlinearity term defined by the following term

$$
\begin{aligned}
& N(q, \dot{q})=B(q) \dot{q} \dot{q}+C(q) \dot{q}^{2}+g(q)= \\
& {\left[\begin{array}{c}
b_{112} \dot{q}_{1} \dot{q}_{2}+b_{113} \dot{q}_{1} \dot{q}_{3}+0+b_{123} \dot{q}_{2} \dot{q}_{3} \\
0+b_{223} \dot{q}_{2} \dot{q}_{3}+0+0 \\
0 \\
b_{412} \dot{q}_{1} \dot{q}_{2}+b_{413} \dot{q}_{1} \dot{q}_{3}+0+0 \\
0 \\
0
\end{array}\right]+\left[\begin{array}{c}
C_{12} \dot{q}_{2}{ }^{2}+C_{13} \dot{q}_{3}{ }^{2} \\
C_{21} \dot{q}_{1}{ }^{2}+C_{23} \dot{q}_{3}{ }^{2} \\
C_{31} \dot{q}_{1}{ }^{2}+C_{32} \dot{q}_{2}{ }^{2} \\
0 \\
C_{51} \dot{q}_{1}{ }^{2}+C_{52} \dot{q}_{2}{ }^{2} \\
0
\end{array}\right]+\left[\begin{array}{c}
0 \\
g_{2} \\
g_{3} \\
0 \\
g_{5} \\
0
\end{array}\right]}
\end{aligned}
$$

Therefore the equation of PD-CTC for control of PUMA 560 robot manipulator is written as the equation of (12);

$$
\begin{aligned}
& {\left[\begin{array}{l}
\widehat{\tau_{1}} \\
\widehat{\tau_{2}} \\
\widehat{\tau_{3}} \\
\widehat{\tau_{4}} \\
\widehat{\tau_{5}} \\
\widehat{\tau_{6}}
\end{array}\right]=} \\
& {\left[\begin{array}{cccccc}
M_{11} & M_{12} & M_{13} & 0 & 0 & 0 \\
M_{21} & M_{22} & M_{23} & 0 & 0 & 0 \\
M_{31} & M_{32} & M_{33} & 0 & M_{35} & 0 \\
\mathbf{0} & 0 & 0 & M_{44} & 0 & 0 \\
\mathbf{0} & \mathbf{0} & \mathbf{0} & \mathbf{0} & M_{55} & 0 \\
\mathbf{0} & \mathbf{0} & \mathbf{0} & \mathbf{0} & \mathbf{0} & M_{66}
\end{array}\right]} \\
& {\left[\begin{array}{l}
\ddot{q}_{d 1}+K_{v 1} \dot{e}_{1}+K_{p 1} e_{1} \\
\ddot{q}_{d 2}+K_{v 2} \dot{e}_{2}+K_{p 2} e_{2} \\
\ddot{q}_{d 3}+K_{v 3} \dot{e}_{3}+K_{p 3} e_{3} \\
\ddot{q}_{d 4}+K_{v 4} \dot{e}_{4}+K_{p 4} e_{4} \\
\ddot{q}_{d 5}+K_{v 5} \dot{e}_{5}+K_{p 5} e_{5} \\
\ddot{q}_{d 6}+K_{v 6} \dot{e}_{6}+K_{p 6} e_{6}
\end{array}\right]+\left[\begin{array}{c}
b_{112} \dot{q}_{1} \dot{q}_{2}+b_{113} \dot{q}_{1} \dot{q}_{3}+0+b_{123} \dot{q}_{2} \dot{q}_{3} \\
0+b_{223} \dot{q}_{2} \dot{q}_{3}+0+0 \\
0 \\
b_{412} \dot{q}_{1} \dot{q}_{2}+b_{413} \dot{q}_{1} \dot{q}_{3}+0+0 \\
0 \\
0
\end{array}\right]} \\
& +\left[\begin{array}{c}
C_{12} \dot{q}_{2}{ }^{2}+C_{13} \dot{q}_{3}{ }^{2} \\
C_{21} \dot{q}_{1}{ }^{2}+C_{23} \dot{q}_{3}{ }^{2} \\
C_{31} \dot{q}_{1}{ }^{2}+C_{32} \dot{q}_{2}^{2} \\
0 \\
C_{51} \dot{q}_{1}^{2}+C_{52} \dot{q}_{2}^{2} \\
0
\end{array}\right]+\left[\begin{array}{c}
0 \\
g_{2} \\
g_{3} \\
0 \\
g_{5} \\
0
\end{array}\right]
\end{aligned}
$$

The controller based on a formulation (12) is related to robot dynamics therefore it has problems in uncertain conditions.

\section{Fuzzy Inference Engine:}

This section provides a review about foundation of fuzzy logic based on [12-13]. Supposed that $U$ is the universe of discourse and $x$ is the element of $U$, therefore, a crisp set can be 
defined as a set which consists of different elements $(x)$ will all or no membership in a set. A fuzzy set is a set that each element has a membership grade, therefore it can be written by the following definition;

$$
\boldsymbol{A}=\left\{\boldsymbol{x}, \boldsymbol{\mu}_{\boldsymbol{A}}(\boldsymbol{x}) \mid \boldsymbol{x} \in \boldsymbol{X}\right\} ; \boldsymbol{A} \in \boldsymbol{U}
$$

Where an element of universe of discourse is $x, \mu_{A}$ is the membership function (MF) of fuzzy set. The membership function $\left(\mu_{A}(x)\right)$ of fuzzy set $A$ must have a value between zero and one. If the membership function $\mu_{A}(x)$ value equal to zero or one, this set change to a crisp set but if it has a value between zero and one, it is a fuzzy set. Defining membership function for fuzzy sets has divided into two main groups; namely; numerical and functional method, which in numerical method each number has different degrees of membership function and functional method used standard functions in fuzzy sets. The membership function which is often used in practical applications includes triangular form, trapezoidal form, bell-shaped form, and Gaussian form. A Trapezoidal membership function of fuzzy set is defined by the following equation

$$
\boldsymbol{\mu}_{\boldsymbol{F}(\boldsymbol{x})}=\left\{\begin{array}{cl}
\mathbf{0}, \boldsymbol{a}, & x<a \\
\frac{\boldsymbol{x}-\boldsymbol{a}}{\boldsymbol{b}-\boldsymbol{x}}, & a \leq x<b \\
\frac{\boldsymbol{d}-\boldsymbol{c}}{\mathbf{0}}, & c \leq x<d \\
\mathbf{0}, & x>d
\end{array}\right.
$$

A Triangular membership function of fuzzy set is defined by the following equation

$$
\boldsymbol{\mu}_{\boldsymbol{F}(\boldsymbol{x})}=\left\{\begin{array}{cl}
\mathbf{0}, \boldsymbol{a} & x<a \\
\frac{\boldsymbol{b}-\boldsymbol{a}}{\boldsymbol{c}-\boldsymbol{x}}, & a \leq x<b \\
\frac{\boldsymbol{c}-\boldsymbol{b}}{\mathbf{0}}, & b \leq x \leq c \\
\mathbf{0}, & x>c
\end{array}\right.
$$

A Gaussian membership function of fuzzy set is defined by

$$
\mu_{F(x)}=e^{\frac{-\left(x-c_{F}\right)^{2}}{W}}
$$

and a Bell-shaped membership function of fuzzy set is defined by

$$
\mu_{F(x)}=\frac{1}{1+\left(x-c_{F}\right)^{2}}
$$




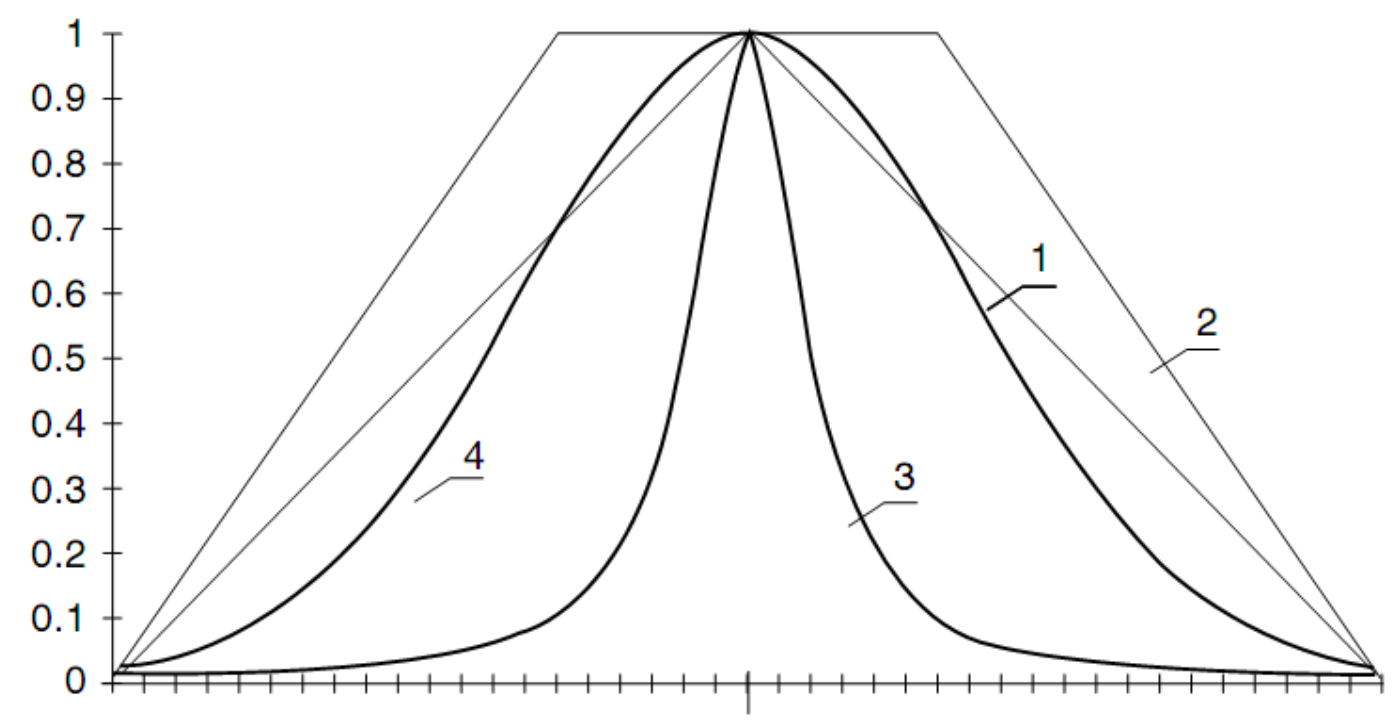

Figure 2. Most Important Membership Functions in Fuzzy Set: 1-Trianglar, 2Trapezoidal, 3-Gaussian, 4-Bell-shaped

Figure 2 shows the typical shapes of membership functions in a fuzzy set.

The union of two fuzzy set $A$ and $B(S-$ norm $)$ is a new fuzzy set which the new membership function is given by

$$
S(a, b)=\mu_{A \cup B(u)}=\max \left\{\mu_{A(u)}, \mu_{B(u)}\right\}, \quad \forall u \in U
$$

The intersection of two fuzzy set $A$ and $B(T-n o r m)$ is a new fuzzy set which the new membership function is given by

$$
\begin{aligned}
& T(a, b)=\mu_{A \cap B(u)}=\min \left\{\mu_{A(u)}, \mu_{B(u)}\right\}=\mu_{A(u)} \cdot \mu_{B(u)} \\
& =\max \left(\mathbf{0}, \mu_{A(u)}+\mu_{B(u)}-\mathbf{1}\right)= \begin{cases}\mu_{A(u)}, & \text { if } \boldsymbol{\mu}_{B(u)}=\mathbf{1} \\
\boldsymbol{\mu}_{B(u)}, & \text { if } \boldsymbol{\mu}_{A(u)}=\mathbf{1} \\
\mathbf{0}, \text { if } \boldsymbol{\mu}_{B(u),}, \boldsymbol{\mu}_{A(u)}<1\end{cases}
\end{aligned}
$$

In fuzzy set the min operation can resolve the statement $A A N D B$ and can be shown by $\min (A, B)$ operation. Using the same reason, the $A O R B$ operation can be replace by max operation in fuzzy set and at last the NOT $A$ operation can be replace by $1-A$ operation in fuzzy set. The algebraic product of two fuzzy set $A$ and $B$ is the multiplication of the membership functions which is given by the following equation

$$
\boldsymbol{\mu}_{A . B(u)}=\boldsymbol{\mu}_{A(u) \cdot} \boldsymbol{\mu}_{B(u)}
$$

The algebraic Sum of two fuzzy sets $A$ and $B$ is given by the following equation

$$
\mu_{A \widehat{+} B(u)}=\mu_{A(u) \cdot} \mu_{B(u)}-\mu_{A(u)} \cdot \mu_{B(u)}
$$


Linguistic variable can open a wide area to use of fuzzy logic theory in many applications (e.g., control and system identification). In a natural artificial language all numbers replaced by words or sentences. In Figure 3 the linguistic variable is torque and the linguistic values are Low,Medium and High.

If - then Rule statements are used to formulate the condition statements in fuzzy logic. A single fuzzy If - then rule can be written by

\section{If $x$ is $A$ Then $y$ is $B$}

where $A$ and $B$ are the Linguistic values that can be defined by fuzzy set, the If - part of the part of " $x$ is $A$ " is called the antecedent part and the then - part of the part of " $y$ is $B$ " is called the Consequent or Conclusion part. The antecedent of a fuzzy if-then rule can have multiple parts, which the following rules shows the multiple antecedent rules:

\section{if $e$ is $N B$ and $\dot{e}$ is $M L$ then $T$ is $L L$}

where $e$ is error, $\dot{e}$ is change of error, $N B$ is Negative Big, $M L$ is Medium Left, $T$ is torque and $L L$ is Large Left.

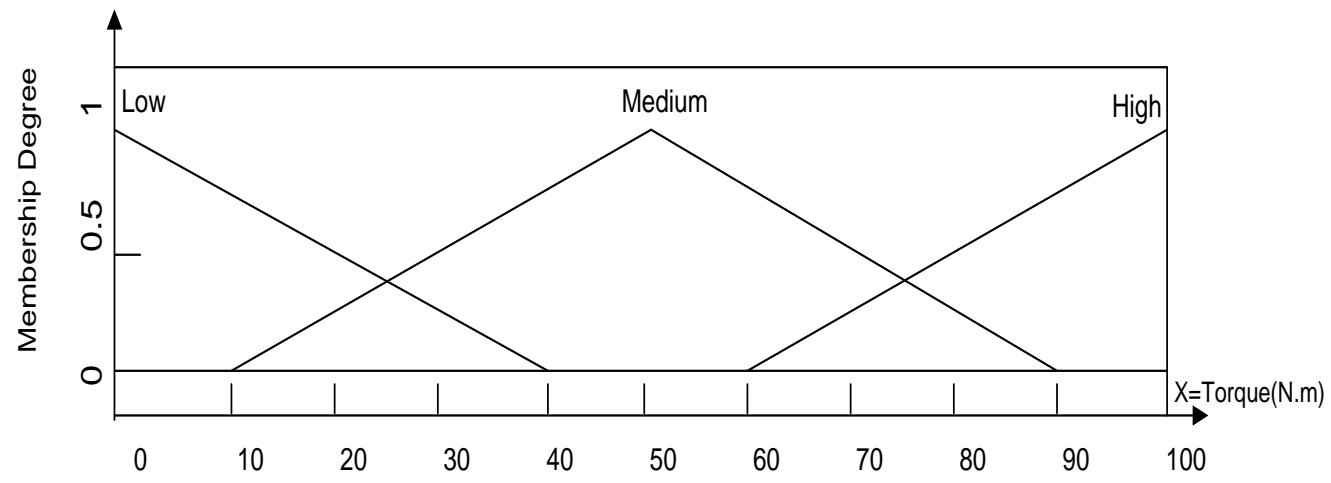

Figure 3. Linguistic Variable and Linguistic Value

If - then rules have three parts, namely, fuzzify inputs, apply fuzzy operator and apply implication method which in fuzzify inputs the fuzzy statements in the antecedent replaced by the degree of membership, apply fuzzy operator used when the antecedent has multiple parts and replaced by single number between 0 to 1 , this part is a degree of support for the fuzzy rule, and apply implication method used in consequent of fuzzy rule to replaced by the degree of membership. Figure 4 shows the main three parts in If - then rules. 


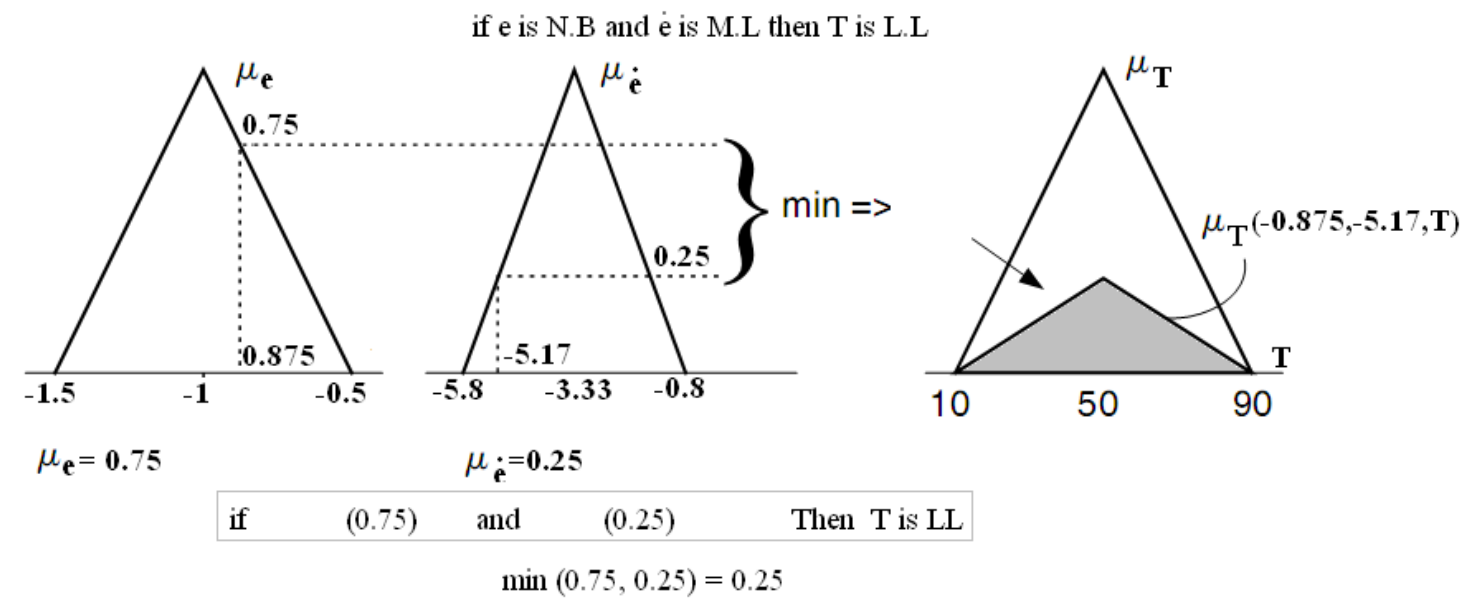

Figure 4. Main Three Parts in IF-THEN Rules in Fuzzy Set

The fuzzy inference engine offers a mechanism for transferring the rule base in fuzzy set which it is divided into two most important methods, namely, Mamdani method and Sugeno method. Mamdani method is one of the common fuzzy inference systems and he designed one of the first fuzzy controllers to control of system engine. Mamdani's fuzzy inference system is divided into four major steps: fuzzification, rule evaluation, aggregation of the rule outputs and defuzzification. Michio Sugeno use a singleton as a membership function of the rule consequent part. The following definition shows the Mamdani and Sugeno fuzzy rule base

$\begin{array}{lllll}\text { Mamdani } & F . R^{1}: \text { if } & x \text { is A and } y \text { is } B & \text { then } & z \text { is } C \\ \text { Sugeno } & F . R^{1}: \text { if } & x \text { is A and } y \text { is } B & \text { then } & f(x, y) \text { is } C\end{array}$

When $x$ and $y$ have crisp values fuzzification calculates the membership degrees for antecedent part. Rule evaluation focuses on fuzzy operation $(A N D / O R)$ in the antecedent of the fuzzy rules. The aggregation is used to calculate the output fuzzy set and several methodologies can be used in fuzzy logic controller aggregation, namely, Max-Min aggregation, Sum-Min aggregation, Max-bounded product, Max-drastic product, Maxbounded sum, Max-algebraic sum and Min-max. Two most common methods that used in fuzzy logic controllers are Max-min aggregation and Sum-min aggregation. Max-min aggregation defined as below

$$
\mu_{U}\left(x_{k}, y_{k}, U\right)=\mu_{\cup_{i=1}^{r} F R^{i}}\left(x_{k}, y_{k}, U\right)=\max \left\{\min _{i=1}^{r}\left[\mu_{R}{ }_{p q}\left(x_{k}, y_{k}\right), \mu_{p_{m}}(U)\right]\right\}
$$

The Sum-min aggregation defined as below

$$
\mu_{U}\left(x_{k}, y_{k}, U\right)=\mu_{\cup_{i=1}^{r} F R^{i}}\left(x_{k}, y_{k}, U\right)=\sum \min _{i=1}^{r}\left[\mu_{R p q}\left(x_{k}, y_{k}\right), \mu_{p_{m}}(U)\right]
$$

where $r$ is the number of fuzzy rules activated by $x_{k}$ and $y_{k}$ and also $\mu_{\mathrm{U}_{i=1}^{r} F R^{i}}\left(x_{k}, y_{k}, U\right)$ is a fuzzy interpretation of $i-t h$ rule. Defuzzification is the last step in the fuzzy inference system which it is used to transform fuzzy set to crisp set. Consequently defuzzification's 
input is the aggregate output and the defuzzification's output is a crisp number. Centre of gravity method (COG) and Centre of area method (COA) are two most common defuzzification methods, which COG method used the following equation to calculate the defuzzification

$$
\operatorname{Cog}\left(x_{k}, y_{k}\right)=\frac{\sum_{i} U_{i} \sum_{j=1}^{r} \cdot \mu_{u}\left(x_{k}, y_{k}, U_{i}\right)}{\sum_{i} \sum_{j=1}^{r} \cdot \mu_{u}\left(x_{k}, y_{k}, U_{i}\right)}
$$

and $C O A$ method used the following equation to calculate the defuzzification

$$
\operatorname{COA}\left(x_{k}, y_{k}\right)=\frac{\sum_{i} U_{i} \cdot \mu_{u}\left(x_{k}, y_{k}, U_{i}\right)}{\sum_{i} \mu_{U} \cdot\left(x_{k}, y_{k}, U_{i}\right)}
$$

Where $\operatorname{COG}\left(x_{k}, y_{k}\right)$ and $\operatorname{COA}\left(x_{k}, y_{k}\right)$ illustrates the crisp value of defuzzification output, $U_{i} \in U$ is discrete element of an output of the fuzzy set, $\mu_{U} .\left(x_{k}, y_{k}, U_{i}\right)$ is the fuzzy set membership function, and $r$ is the number of fuzzy rules.

Based on foundation of fuzzy logic methodology; fuzzy logic controller has played important rule to design nonlinear controller for nonlinear and uncertain systems [53]. However the application area for fuzzy control is really wide, the basic form for all command types of controllers consists of;

- Input fuzzification (binary-to-fuzzy[B/F]conversion)

- Fuzzy rule base (knowledge base)

- Inference engine

- Output defuzzification (fuzzy-to-binary[F/B]conversion).

The basic structure of a fuzzy controller is shown in Figure 5.

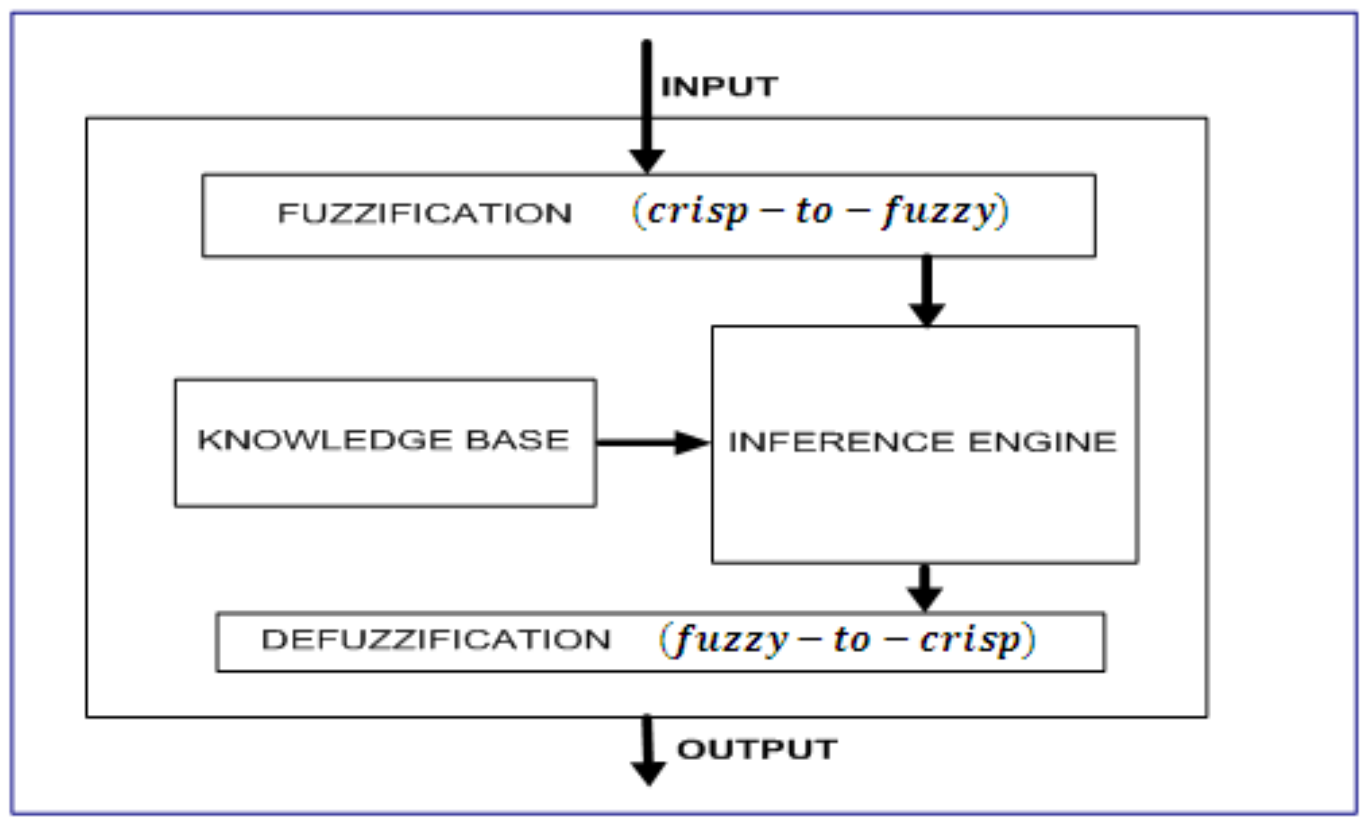

Figure 5. Structure of Fuzzy Logic Controller (FLC) 
Based on Figure 5; fuzzification is used to change the crisp set into fuzzy set. Knowledge base is used to rule evaluation and determine the membership degree and if all fuzzy inputs activated by the known input values. Fuzzy inference engine is used to transferring the rule base into fuzzy set by Mamdani's or Sugeno method based on aggregation of the rules output. Deffuzification is the last part to calculate the fuzzy inference system.

\section{Methodology}

This part is focused on design SISO computed torque like methodology to resolve uncertain problem. The firs type of fuzzy systems is given by

$$
\begin{aligned}
& \operatorname{COA}\left(x_{k}, y_{k}\right)=\frac{\sum_{i} U_{i} \cdot \mu_{u}\left(x_{k}, y_{k}, U_{i}\right)}{\sum_{i} \mu_{U^{*}}\left(x_{k}, y_{k}, U_{i}\right)} \\
& f(x)=\sum_{l=1}^{M} \theta^{l} \mathcal{E}^{l}(x)=\theta^{T} \mathcal{E}(x)
\end{aligned}
$$

Where

$\theta=\left(\theta^{1}, \ldots, \theta^{M}\right)^{T}, \mathcal{E}(x)=\left(\mathcal{E}^{1}(x), \ldots, \mathcal{E}^{M}(x)\right)^{T}$, and $\mathcal{E}^{l}(x)=$

$: \prod_{i=1}^{n} \frac{{ }_{A_{i}^{l}}\left(x_{i}\right)}{\sum_{l=1}^{M}}\left(\prod_{i=1}^{n} \mu_{A_{i}^{l}}\left(x_{i}\right)\right) . \quad \theta^{1}, \ldots, \theta^{M}$ are adjustable parameters in (29) . $\mu_{A_{1}^{1}}\left(x_{1}\right), \ldots, \mu_{A_{n}^{m}}\left(x_{n}\right)$ are given membership functions whose parameters will not change over time.

The second type of fuzzy systems is given by

$$
f(x)=\frac{\sum_{l=1}^{M} \theta^{l}\left[\prod_{i=1}^{n} \exp \left(-\left(\frac{x_{i}-\alpha_{i}^{l}}{\delta_{i}^{l}}\right)^{2}\right)\right]}{\sum_{l=1}^{M}\left[\prod_{i=1}^{n} \exp \left(-\left(\frac{x_{i}-\alpha_{i}^{l}}{\delta_{i}^{l}}\right)^{2}\right)\right]}
$$

Where $\theta^{l}, \alpha_{i}^{l}$ and $\delta_{i}^{l}$ are all adjustable parameters. From the universal approximation theorem, we know that we can find a fuzzy system to estimate any continuous function. For the first type of fuzzy systems, we can only adjust $\theta^{l}$ in (24). We define $f^{\wedge}(x \mid \theta)$ as the approximator of the real function $f(x)$.

$$
f^{\wedge}(x \mid \theta)=\theta^{T} \varepsilon(x)
$$

We define $\theta^{*}$ as the values for the minimum error:

$$
\theta^{*}=\arg \min _{\theta \in \Omega}\left[\sup _{x \in U}\left|f^{\wedge}(x \mid \theta)-g(x)\right|\right]
$$

Where $\Omega$ is a constraint set for $\theta$. For specific $x, \sup _{x \in U}\left|f^{\wedge}\left(x \mid \theta^{*}\right)-f(x)\right|$ is the minimum approximation error we can get.

We used the first type of fuzzy systems (29) to estimate the nonlinear system (11) the fuzzy formulation can be write as below; 


$$
f(x \mid \theta)=\theta^{T} \varepsilon(x)=\frac{\sum_{l=1}^{n} \theta^{l}\left[\mu_{A^{l}}(x)\right]}{\sum_{l=1}^{n}\left[\mu_{A^{l}}(x)\right]}
$$

Where $\theta^{1}, \ldots, \theta^{n}$ are adjusted by an adaptation law. The general SISO if-then rules are given by

$$
\begin{gathered}
R^{l} \text { : if } x_{1} \text { is } A_{1}^{l}, x_{2} \text { is } A_{2}^{l}, \ldots, x_{n} \text { is } A_{n}^{l} \\
\text { then } y_{1} \text { is } B_{1}^{l}, \ldots, y_{m} \text { is } B_{m}^{l}
\end{gathered}
$$

Where $l=1,2, \ldots, M$ are fuzzy if-then rules; $x=\left(x_{1}, \ldots, x_{n}\right)^{T}$ and $y=\left(y_{1}, \ldots, y_{n}\right)^{T}$ are the input and output vectors of the fuzzy system. The SISO fuzzy system is define as

$$
f(x)=\ominus^{T} \varepsilon(x)
$$

Where

$$
\ominus^{T}=\left(\theta_{1}, \ldots, \theta_{m}\right)^{T}=\left[\begin{array}{c}
\theta_{1}^{1}, \theta_{1}^{2}, \ldots, \theta_{1}^{M} \\
\theta_{2}^{1}, \theta_{2}^{2}, \ldots, \theta_{2}^{M} \\
\vdots \\
\theta_{m}^{1}, \theta_{m}^{2}, \ldots, \boldsymbol{\theta}_{m}^{M}
\end{array}\right]
$$

$\varepsilon(x)=\left(\varepsilon^{1}(x), \ldots, \varepsilon^{M}(x)\right)^{T}, \quad \varepsilon^{1}(x)=\prod_{i=1}^{n} \mu_{A_{i}^{l}}\left(x_{i}\right) / \sum_{l=1}^{M}\left(\prod_{i=1}^{n} \mu_{A_{i}^{l}}\left(x_{i}\right)\right), \quad$ and $\mu_{A_{i}^{l}}\left(x_{i}\right)$ is defined in (33). To reduce the number of fuzzy rules, we divide the fuzzy system in to three parts:

$$
\begin{aligned}
F^{1}(q, \dot{q}) & =\ominus^{1^{T}} \varepsilon(q, \dot{q}) \\
& =\left[\theta_{1}^{1^{T}} \varepsilon(q, \dot{q}), \ldots, \theta_{m}^{1^{T}} \varepsilon(q, \dot{q})\right]^{T} \\
F^{2}\left(q, \ddot{q}_{r}\right) & =\ominus^{2^{T}} \varepsilon\left(q, \ddot{q}_{r}\right)=\left[\theta_{1}^{2^{T}} \varepsilon\left(q, \ddot{q}_{r}\right), \ldots,{\theta_{m}^{2}}^{T} \varepsilon\left(q, \ddot{q}_{r}\right)\right]^{T} \\
F^{3}(q, \ddot{q}) & =\ominus^{3^{T}} \varepsilon(q, \ddot{q}) \\
& =\left[\theta_{1}^{3^{T}} \varepsilon(q, \dot{q}), \ldots,{\theta_{m}^{3}}^{T} \varepsilon(q, \ddot{q})\right]^{T}
\end{aligned}
$$

The control security input is given by

$$
\begin{aligned}
& \tau=M^{\wedge} \ddot{q}_{r}+B(q) \dot{q} \dot{q}+C(q) \dot{q}^{2}+g(q)+F^{1}(q, \dot{q})+F^{2}\left(q, \ddot{q}_{r}\right)+F^{3}(q, \ddot{q})- \\
& K_{p} e-K_{v} \dot{e}
\end{aligned}
$$

Where $M^{\wedge}, \boldsymbol{B}(\boldsymbol{q}) \dot{\boldsymbol{q}} \dot{\boldsymbol{q}}, \boldsymbol{C}(\boldsymbol{q}) \dot{\boldsymbol{q}}^{2}, \boldsymbol{g}(\boldsymbol{q})$ are the estimations of $M(q)$.

Consider a nonlinear single input dynamic system is defined by: 


$$
x^{(n)}=f(\vec{x})+b(\vec{x}) u
$$

Where $\mathrm{u}$ is the vector of control input, $\boldsymbol{x}^{(\boldsymbol{n})}$ is the $\boldsymbol{n}^{\text {th }}$ derivation of $\boldsymbol{x}$, $\boldsymbol{x}=\left[\boldsymbol{x}, \dot{\boldsymbol{x}}, \ddot{\boldsymbol{x}}, \ldots, \boldsymbol{x}^{(\boldsymbol{n}-1)}\right]^{\boldsymbol{T}}$ is the state vector, $\boldsymbol{f}(\boldsymbol{x})$ is unknown or uncertainty, and $\boldsymbol{b}(\boldsymbol{x})$ is of known sign function. The main goal to design this controller is train to the desired state; $\boldsymbol{x}_{\boldsymbol{d}}=\left[\boldsymbol{x}_{\boldsymbol{d}}, \dot{\boldsymbol{x}}_{\boldsymbol{d}}, \ddot{\boldsymbol{x}}_{\boldsymbol{d}}, \ldots, \boldsymbol{x}_{\boldsymbol{d}}^{(\boldsymbol{n}-1)}\right]^{\boldsymbol{T}}$, and trucking error vector is defined by:

$$
\widetilde{x}=x-x_{d}=\left[\widetilde{x}, \ldots, \widetilde{x}^{(n-1)}\right]^{T}
$$

A time-varying sliding surface $\boldsymbol{s}(\boldsymbol{x}, \boldsymbol{t})$ in the state space $\boldsymbol{R}^{\boldsymbol{n}}$ is given by:

$$
s(x, t)=\left(\frac{d}{d t}+\lambda\right)^{n-1} \widetilde{x}=0
$$

where $\lambda$ is the positive constant. To further penalize tracking error, integral part can be used in sliding surface part as follows:

$$
s(x, t)=\left(\frac{d}{d t}+\lambda\right)^{n-1}\left(\int_{0}^{t} \widetilde{x} d t\right)=0
$$

The main target in this methodology is kept the sliding surface slope $\boldsymbol{s}(\boldsymbol{x}, \boldsymbol{t})$ near to the zero. Therefore, one of the common strategies is to find input $\boldsymbol{U}$ outside of $\boldsymbol{s}(\boldsymbol{x}, \boldsymbol{t})$.

$$
\frac{1}{2} \frac{d}{d t} s^{2}(x, t) \leq-\zeta|s(x, t)|
$$

where $\zeta$ is positive constant.

$$
\text { If } \mathbf{S}(\mathbf{0})>0 \rightarrow \frac{\mathrm{d}}{\mathrm{dt}} \mathbf{S}(\mathrm{t}) \leq-\zeta
$$

To eliminate the derivative term, it is used an integral term from $t=0$ to $t=t_{\text {reach }}$

$$
\int_{t=0}^{t=t_{\text {reach }}} \frac{d}{d t} S(t) \leq-\int_{t=0}^{t=t_{\text {reach }}} \eta \rightarrow S\left(t_{\text {reach }}\right)-S(0) \leq-\zeta\left(t_{\text {reach }}-0\right)
$$

Where $t_{\text {reach }}$ is the time that trajectories reach to the sliding surface so, suppose $\mathrm{S}\left(t_{\text {reach }}=0\right)$ defined as

$$
0-S(0) \leq-\eta\left(t_{\text {reach }}\right) \rightarrow t_{\text {reach }} \leq \frac{S(0)}{\zeta}
$$

And

$$
\text { if } S(0)<0 \rightarrow 0-S(0) \leq-\eta\left(t_{\text {reach }}\right) \rightarrow S(0) \leq-\zeta\left(t_{\text {reach }}\right) \rightarrow t_{\text {reach }} \leq \frac{|S(0)|}{\eta}
$$


Equation (50) guarantees time to reach the sliding surface is smaller than $\frac{|\boldsymbol{S}(\mathbf{0})|}{\zeta}$ since the trajectories are outside of $S(t)$.

$$
\text { if } S_{t_{\text {reach }}}=S(0) \rightarrow \operatorname{error}\left(x-x_{d}\right)=0
$$

suppose $\mathrm{S}$ is defined as

$$
s(x, t)=\left(\frac{d}{d t}+\lambda\right) \quad \tilde{x}=\left(\dot{\mathbf{x}}-\dot{\mathbf{x}}_{\mathbf{d}}\right)+\lambda\left(\mathbf{x}-\mathbf{x}_{\mathbf{d}}\right)
$$

The derivation of S, namely, $\dot{S}$ can be calculated as the following;

$$
\dot{\boldsymbol{S}}=\left(\ddot{\mathbf{x}}-\ddot{\mathbf{x}}_{\mathbf{d}}\right)+\lambda\left(\dot{\mathbf{x}}-\dot{\mathbf{x}}_{\mathbf{d}}\right)
$$

suppose the second order system is defined as;

$$
\ddot{\boldsymbol{x}}=\boldsymbol{f}+\boldsymbol{u} \rightarrow \dot{S}=\boldsymbol{f}+\boldsymbol{U}-\ddot{\boldsymbol{x}}_{\boldsymbol{d}}+\lambda\left(\dot{\mathbf{x}}-\dot{\mathbf{x}}_{\mathbf{d}}\right)
$$

Where $f$ is the dynamic uncertain, and also since $S=0$ and $\dot{S}=0$, to have the best approximation, $\widehat{\boldsymbol{U}}$ is defined as

$$
\widehat{U}=-\widehat{f}+\ddot{x}_{d}-\lambda\left(\dot{\mathbf{x}}-\dot{\mathbf{x}}_{\mathbf{d}}\right)
$$

A simple solution to get the sliding condition when the dynamic parameters have uncertainty is the switching control law:

$$
U_{\text {switching }}=\widehat{U}-K(\vec{x}, t) \cdot \operatorname{sgn}(s)
$$

where the switching function $\mathbf{s g n}(\mathbf{S})$ is defined as

$$
\operatorname{sgn}(s)= \begin{cases}1 & s>0 \\ -1 & s<0 \\ 0 & s=0\end{cases}
$$

and the $\boldsymbol{K}(\overrightarrow{\boldsymbol{x}}, \boldsymbol{t})$ is the positive constant. Suppose by (45) the following equation can be written as,

$$
\frac{1}{2} \frac{d}{d t} s^{2}(x, t)=\dot{S} \cdot S=[f-\widehat{f}-K \operatorname{sgn}(s)] \cdot S=(f-\widehat{f}) \cdot S-K|S|
$$

and if the equation (50) instead of (49) the sliding surface can be calculated as

$$
s(x, t)=\left(\frac{d}{d t}+\lambda\right)^{2}\left(\int_{0}^{t} \widetilde{x} d t\right)=\left(\dot{\mathbf{x}}-\dot{\mathbf{x}}_{\mathrm{d}}\right)+2 \lambda\left(\dot{\mathrm{x}}-\dot{\mathbf{x}}_{\mathrm{d}}\right)-\lambda^{2}\left(\mathrm{x}-\mathrm{x}_{\mathrm{d}}\right)
$$


where $S=\lambda e+\dot{e}$ in PD-SMC and $S=\lambda e+\dot{e}+\left(\frac{\lambda}{2}\right)^{2} \sum e$ in PID-SMC. The adaptation law is given by

$$
\begin{aligned}
\dot{\theta}_{j}^{1} & =-\Gamma_{1 j} s_{j} \varepsilon(q, \dot{q}) \\
\dot{\theta}_{j}^{2} & =-\Gamma_{2 j} s_{j} \varepsilon\left(q, \ddot{q}_{r}\right) \\
\dot{\theta}_{j}^{3} & =-\Gamma_{3 j} s_{j} \varepsilon(q, \ddot{q})
\end{aligned}
$$

Where $j=1, \ldots, m$ and $\Gamma_{1 j}-\Gamma_{3 j}$ are positive diagonal matrices.

The Lyapunov function candidate is presented as

$$
V=\frac{1}{2} s^{T} M s+\frac{1}{2} \sum_{j=1}^{m} \frac{1}{\Gamma_{1 j}} \emptyset_{j}^{1^{T}} \emptyset_{j}^{1}+\frac{1}{2} \sum_{j=1}^{m} \frac{1}{\Gamma_{2 j}} \phi_{j}^{2^{T}} \emptyset_{j}^{2}+\frac{1}{2} \sum_{j=1}^{m} \frac{1}{\Gamma_{3 j}} \emptyset_{j}^{13^{T}} \emptyset_{j}^{3}
$$

Where $\emptyset_{j}^{1}=\emptyset_{j}^{1^{*}}-\emptyset_{j}^{1}, \emptyset_{j}^{2}=\emptyset_{j}^{2^{*}}-\emptyset_{j}^{2}$ and $\emptyset_{j}^{3}=\emptyset_{j}^{3^{*}}-\emptyset_{j}^{3}$ we define

$$
F\left(q, \dot{q}, \ddot{q}_{r}, \ddot{q}\right)=F^{1}(q, \dot{q})+F^{2}\left(q, \ddot{q}_{r}\right)+F^{3}(q, \ddot{q})
$$

From (27) and (28), we get

$$
\begin{aligned}
& M(q) \ddot{q}+B(q) \dot{q} \dot{q}+C(q) \dot{q}^{2}+g(q)=M^{\wedge} \ddot{q}_{r}+B(q) \dot{q} \dot{q}+\widehat{C(q)} \dot{q}^{2}+g(q)+ \\
& F\left(q, \dot{q}, \ddot{q}_{r}, \ddot{q}\right)-K_{D} s-W s g n(s)
\end{aligned}
$$

Since $\dot{q}_{r}=\dot{q}-s$ and $\ddot{q}_{r}=\ddot{q}-\dot{s}$, we get

$$
M \dot{s}+\left(B(q) \dot{q} \dot{q}+C(q) \dot{q}^{2}+g(q)+K_{D}\right) s+W s g n(s)=-\Delta F+F\left(q, \dot{q}, \ddot{q}_{r}, \ddot{q}\right)
$$

Then $M \dot{s}+\boldsymbol{B}(\boldsymbol{q}) \dot{\boldsymbol{q}} \dot{\boldsymbol{q}}+\boldsymbol{C}(\boldsymbol{q}) \dot{\boldsymbol{q}}^{2}+\boldsymbol{g}(\boldsymbol{q}) s$ can be written as

$$
M \dot{s}+B(q) \dot{q} \dot{q}+C(q) \dot{q}^{2}+g(q) s=-\Delta F+F\left(q, \dot{q}, \ddot{q}_{r}, \ddot{q}\right)-K_{D} s-W \operatorname{sgn}(s)
$$

Where $\Delta F=\widetilde{M} \ddot{q}_{r}+\boldsymbol{B}(\boldsymbol{q}) \dot{\boldsymbol{q}} \dot{\boldsymbol{q}}+\boldsymbol{C}(\boldsymbol{q}) \dot{\boldsymbol{q}}^{2}+\boldsymbol{g}(\boldsymbol{q}), \widetilde{M}=M-M^{\wedge}, \tilde{C}_{1}=\boldsymbol{B}(\boldsymbol{q}) \dot{\boldsymbol{q}} \dot{\boldsymbol{q}}+\boldsymbol{C}(\boldsymbol{q}) \dot{\boldsymbol{q}}^{2}+$ $\boldsymbol{g}(\boldsymbol{q})-\boldsymbol{B}(\boldsymbol{q}) \dot{\boldsymbol{q}} \dot{\boldsymbol{q}}+\widehat{\boldsymbol{C}(\boldsymbol{q})} \dot{\boldsymbol{q}}^{2}+\boldsymbol{g}(\boldsymbol{q})$ The derivative of $V$ is

$$
\dot{V}=s^{T} M \dot{s}+\frac{1}{2} s^{T} \dot{M} s+\sum_{j=1}^{m} \frac{1}{\Gamma_{1 j}} \emptyset_{j}^{1^{T}} \dot{\emptyset}_{j}^{1}+\sum_{j=1}^{m} \frac{1}{\Gamma_{2 j}} \emptyset_{j}^{2^{T}} \dot{\emptyset}_{j}^{2}+\sum_{j=1}^{m} \frac{1}{\Gamma_{3 j}} \emptyset_{j}^{13^{T}} \dot{\emptyset}_{j}^{3}
$$

We know that $s^{T} M \dot{s}+\frac{1}{2} s^{T} \dot{M} s=s^{T}\left(M \dot{s}+\boldsymbol{B}(\boldsymbol{q}) \dot{\boldsymbol{q}} \dot{\boldsymbol{q}}+\boldsymbol{C}(\boldsymbol{q}) \dot{\boldsymbol{q}}^{2}+\boldsymbol{g}(\boldsymbol{q}) s\right)$ from (66). Then

$$
\begin{aligned}
& \dot{V}=-s^{T}\left[-K_{D} s+W \operatorname{sgn}(s)+\Delta F-F\left(q, \dot{q}, \ddot{q}_{r}, \ddot{q}\right)\right]+\sum_{j=1}^{m} \frac{1}{\Gamma_{1 j}} \emptyset_{j}^{1^{T}} \dot{\phi}_{j}^{1}+ \\
& \sum_{j=1}^{m} \frac{1}{\Gamma_{2 j}} \phi_{j}^{2^{T}} \dot{\phi}_{j}^{2}+\sum_{j=1}^{m} \frac{1}{\Gamma_{3 j}} \emptyset_{j}^{13^{T}} \dot{\phi}_{j}^{3}
\end{aligned}
$$

We define the minimum approximation error as 


$$
\omega=\Delta \boldsymbol{F}-\left[\boldsymbol{F}^{\mathbf{1}}\left(\boldsymbol{q}, \dot{\boldsymbol{q}} \mid \ominus^{\mathbf{1}^{*}}\right)+\boldsymbol{F}^{\mathbf{2}}\left(\boldsymbol{q}, \ddot{\boldsymbol{q}}_{r} \mid \ominus^{2^{*}}\right)+\boldsymbol{F}^{\mathbf{3}}\left(\boldsymbol{q}, \ddot{\boldsymbol{q}} \mid \ominus^{3^{*}}\right)\right]
$$

We plug (67) in to (68)

$$
\begin{aligned}
& \dot{V}=-s^{T}\left[-K_{D} s+W \operatorname{sgn}(s)+\Delta F-F\left(q, \dot{q}, \ddot{q}_{r}, \ddot{q}\right)\right]+ \\
& \sum_{j=1}^{m} \frac{1}{\Gamma_{1 j}} \phi_{j}^{1^{T}} \dot{\emptyset}_{j}^{1}+\sum_{j=1}^{m} \frac{1}{\Gamma_{2 j}} \phi_{j}^{2^{T}} \dot{\emptyset}_{j}^{2}+\sum_{j=1}^{m} \frac{1}{\Gamma_{3 j}} \phi_{j}^{13^{T}} \dot{\phi}_{j}^{3} \\
& = \\
& -s^{T}\left[-K_{D} s+W \operatorname{sgn}(s)+\omega+F^{1}\left(q, \dot{q} \mid \ominus^{1^{*}}\right)+F^{2}\left(q, \ddot{q}_{r} \mid \ominus^{2^{*}}\right)+\right. \\
& \left.F^{3}\left(q, \ddot{q} \mid \ominus^{3^{*}}\right)-F^{1}(q, \dot{q})+F^{2}\left(q, \ddot{q}_{r}\right)+F^{3}(q, \ddot{q})\right]+\sum_{j=1}^{m} \frac{1}{\Gamma_{1 j}} \phi_{j}^{1^{T}} \dot{\phi}_{j}^{1}+ \\
& \sum_{j=1}^{m} \frac{1}{\Gamma_{2 j}} \phi_{j}^{2^{T}} \dot{\phi}_{j}^{2}+\sum_{j=1}^{m} \frac{1}{\Gamma_{3 j}} \phi_{j}^{13^{T}} \dot{\phi}_{j}^{3} \\
& =-s^{T} K_{D} s-s^{T} W \operatorname{sgn}(s)-s^{T} \omega-\sum_{j=1}^{m} s_{j} \phi_{j}^{1^{T}} \varepsilon(q, \dot{q})-\sum_{j=1}^{m} s_{j} \phi_{j}^{2^{T}} \varepsilon\left(q, \ddot{q}_{r}\right)- \\
& \sum_{j=1}^{m} s_{j} \phi_{j}^{3^{T}} \varepsilon(q, \ddot{q})+\sum_{j=1}^{m} \frac{1}{\Gamma_{1 j}} \phi_{j}^{1^{T}} \dot{\emptyset}_{j}^{1}+\sum_{j=1}^{m} \frac{1}{\Gamma_{2 j}} \phi_{j}^{2^{T}} \dot{\phi}_{j}^{2}+\sum_{j=1}^{m} \frac{1}{\Gamma_{3 j}} \phi_{j}^{3^{T}} \dot{\phi}_{j}^{3} \\
& =-s^{T} K_{D} s-s^{T} W \operatorname{sgn}(s)-s^{T} \omega-\sum_{j=1}^{m} \phi_{j}^{1^{T}}\left(s_{j} \varepsilon(q, \dot{q})-\frac{1}{\Gamma_{1 j}} \dot{\phi}_{j}^{1}\right)- \\
& \sum_{j=1}^{m} \phi_{j}^{2^{T}}\left(s_{j} \varepsilon\left(q, \ddot{q}_{r}\right)-\frac{1}{\Gamma_{2 j}} \dot{\phi}_{j}^{2}\right)-\sum_{j=1}^{m} \emptyset_{j}^{3^{T}}\left(s_{j} \varepsilon\left(q, \ddot{q}_{r}\right)-\frac{1}{\Gamma_{3 j}} \dot{\phi}_{j}^{3}\right) \\
& =-s^{T} K_{D} s-s^{T} W \operatorname{sgn}(s)-s^{T} \omega-\sum_{j=1}^{m} \emptyset_{j}^{1^{T}}\left(s_{j} \varepsilon(q, \dot{q})+\frac{1}{\Gamma_{1 j}} \dot{\phi}_{j}^{1}\right)- \\
& \sum_{j=1}^{m} \emptyset_{j}^{2^{T}}\left(s_{j} \varepsilon\left(q, \ddot{q}_{r}\right)+\frac{1}{\Gamma_{2 j}} \dot{\phi}_{j}^{2}\right)-\sum_{j=1}^{m} \phi_{j}^{3^{T}}\left(s_{j} \varepsilon\left(q, \ddot{q}_{r}\right)+\frac{1}{\Gamma_{3 j}} \dot{\phi}_{j}^{3}\right)
\end{aligned}
$$

Then $\dot{V}$ becomes

$$
\begin{aligned}
\dot{V} & =-s^{T} K_{D} s-s^{T} W \operatorname{sgn}(s)-s^{T} \omega \\
& =-\sum_{j=1}^{m}\left(s_{j}^{2} K_{D j}+W_{j}\left|s_{j}\right|+s_{j} \omega_{j}\right) \\
& =-\sum_{j=1}^{m}\left[s_{j}\left(s_{j} K_{D j}+\omega_{j}\right)+W_{j}\left|s_{j}\right|\right]
\end{aligned}
$$

Since $\omega_{j}$ can be as small as possible, we can find $K_{D j}$ that $\left|s_{j}^{2} K_{D j}\right|>\left|\omega_{j}\right|\left(s_{j} \neq 0\right)$.

Therefore, we can get $s_{j}\left(s_{j} K_{D j}+\omega_{j}\right)>0$ for $s_{j} \neq 0$ and $\dot{V}<0(s \neq 0)$.

\section{Results}

To test the noise rejection safety in nonlinear uncertain system in presence of external disturbance computed torque like method (CTLC) and partly sliding switching computed torque like method (ACTLC) are compared. In this research trajectory following are compared in these two methods because it is very important to show the safety method in industrial factory.

Trajectory: Figure 6 shows the links trajectory in CTLC and proposed SISO ACTLC without disturbance for sinus trajectory in general and zoom scaling because all 3 links have the same response so, all links are shown in a graph. 


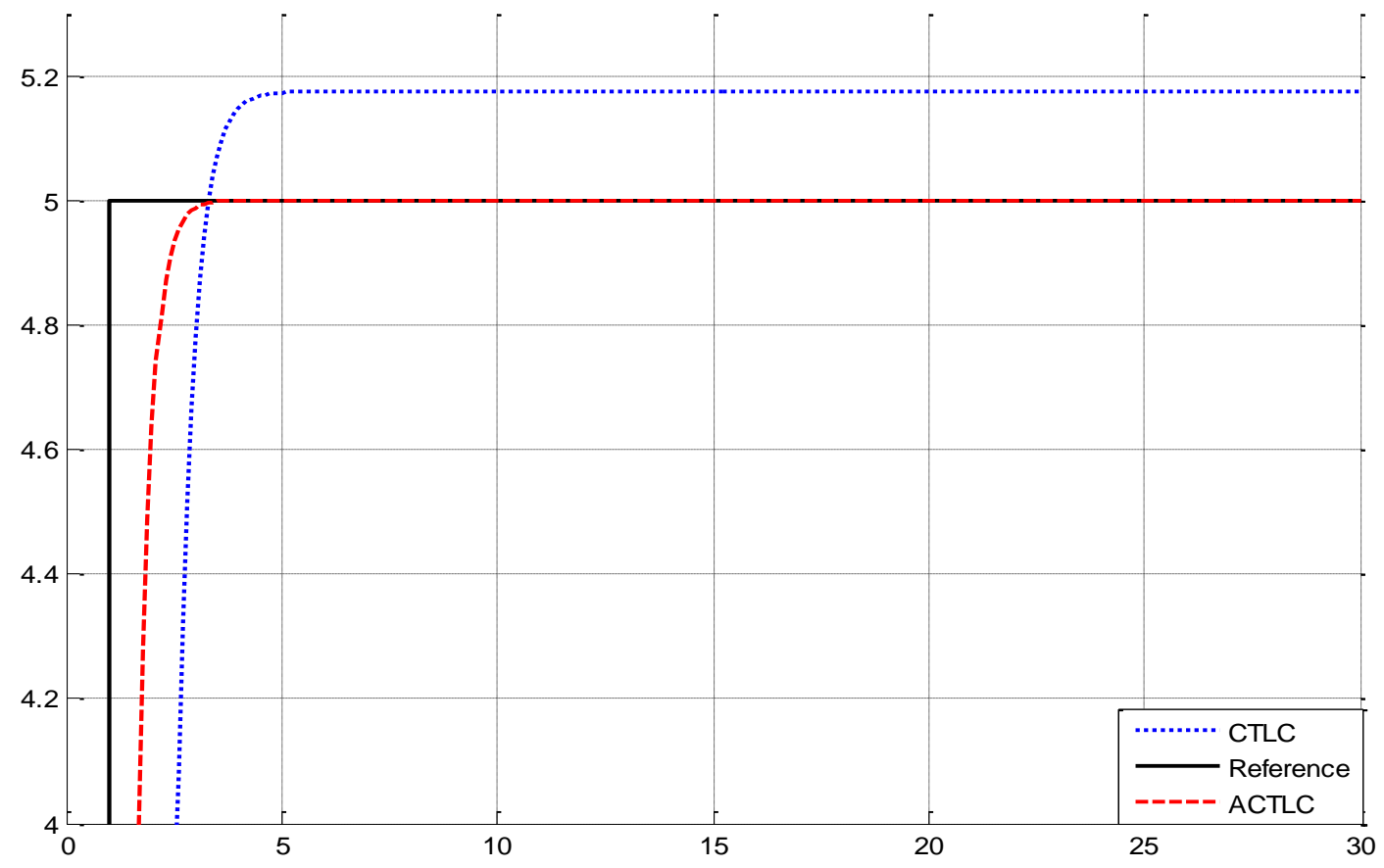

Figure 6. CTLC Vs ACTLC

By comparing sinus response, Figure 6, in two methods, however both methods have acceptable performance but ACTLC's error about zero but CTLC has a fixed error.

Disturbance rejection: Figure 7 shows the power disturbance removal in CTLC and ACTLC. Besides a band limited white noise with predefined of $40 \%$ the power of input signal is applied to the sinus CTLC and ACTLC; it found slight oscillations in CTLC trajectory responses. All 3 links are shown in one graph. 


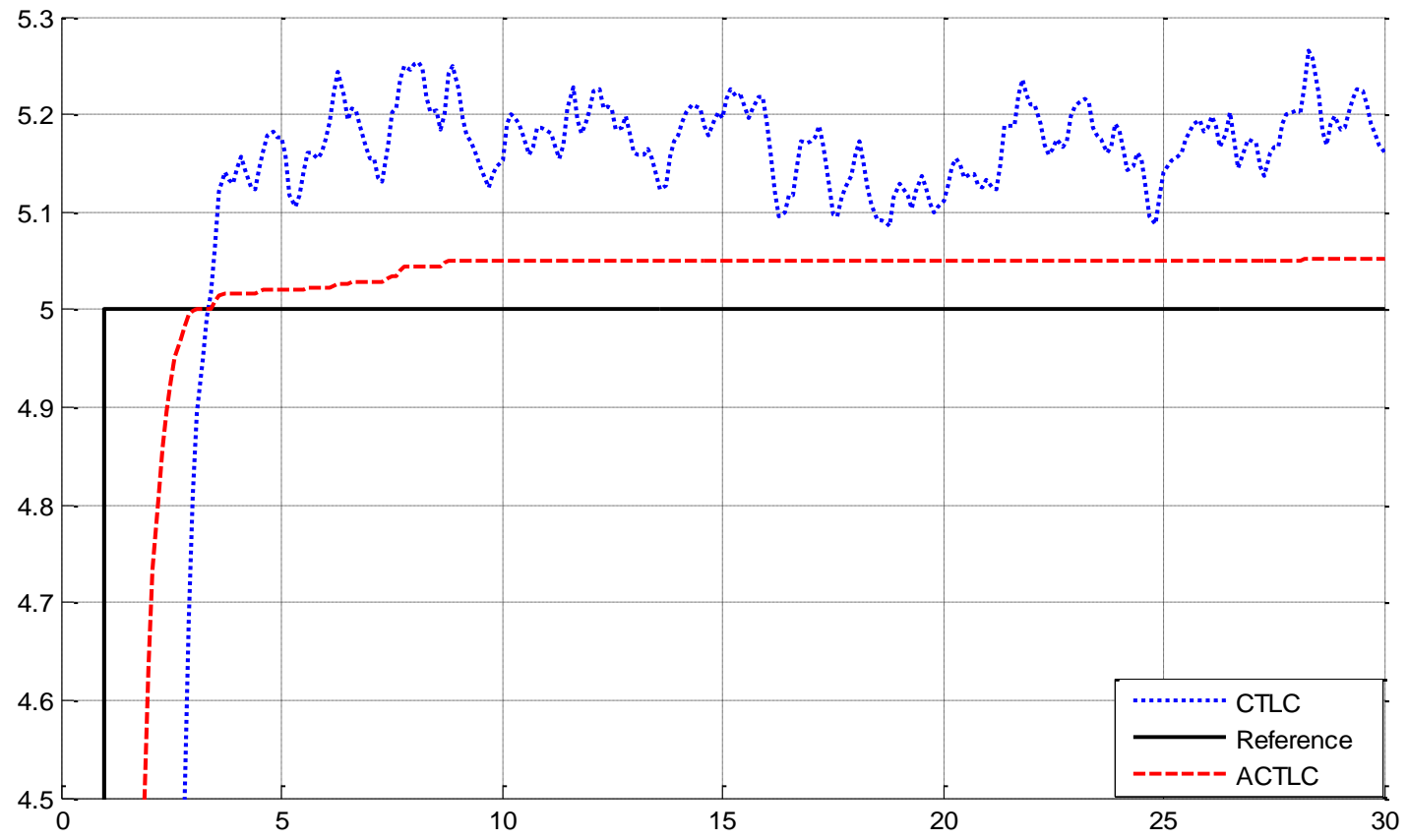

Figure 7. CTLC Vs ACTLC: Uncertain Robot arm with External Disturbance

Among above graph, relating to external disturbance, CTLC has slightly fluctuations. Regarding to Figure 7, CTLC is robust less to solve this challenge ACTLC is introduced. in presence of uncertainty and external disturbances, ACTLC has steady errors compared to certain condition but it is more robust than CTLC.

\section{Conclusion}

Refer to the research, a secure adaptive partly sliding switching computed torque like method based on artificial intelligence methodology has proposed in order to design safety high performance nonlinear controller in the presence of uncertainties and external disturbances. Regarding to the positive points in computed torque methodology, fuzzy inference system and adaptive partly sliding switching methodology it is found that the adaptation laws derived in the system to resolve the uncertainty and external disturbance to reach the safe environment. The safety stability of the closed-loop system is proved mathematically based on the Lyapunov method. The first target in this work is compensate the model uncertainty by SISO fuzzy inference system, in the case of robot arm, if we define $\boldsymbol{k}_{\mathbf{1}}$ membership functions for each input variable, the number of fuzzy rules applied for each joint is $\boldsymbol{K}_{\mathbf{1}}$ which will result in a low computational load. In second target partly sliding switching methodology is used to online tuning and adjusted the computed torque like method to eliminate the overshoot in industrial robot arm which this problem is caused to have unsecured environment. In this case the performance is improved by using the advantages of computed torque, artificial intelligence compensate method and adaptive algorithm while the disadvantages removed by added each method to previous method. 


\section{Acknowledgments}

The authors would like to thank the anonymous reviewers for their careful reading of this paper and for their helpful comments. This work was supported by the Iranian Institute of Advance Science and Technology Program of Iran under grant no. 2012-Persian Gulf-2A.

Iranian center of Advance Science and Technology (IRAN SSP) is one of the independent research centers specializing in research and training across of Control and Automation, Electrical and Electronic Engineering, and Mechatronics \& Robotics in Iran. At IRAN SSP research center, we are united and energized by one mission to discover and develop innovative engineering methodology that solve the most important challenges in field of advance science and technology. The IRAN SSP Center is instead to fill a long standing void in applied engineering by linking the training a development function one side and policy research on the other. This center divided into two main units:

- $\quad$ Education unit

- $\quad$ Research and Development unit

\section{References}

[1] T. R. Kurfess, Robotics and automation handbook: CRC, 2005.

[2] J. J. E. Slotine and W. Li, "Applied nonlinear control vol. 461: Prentice hall Englewood Cliffs", NJ, (1991).

[3] F. Piltan, M. Akbari, M. Piran and M. Bazregar, "Design Model Free Switching Gain Scheduling Baseline Controller with Application to Automotive Engine", International Journal of Information Technology and Computer Science, 01:65-73, (2013).

[4] F. Piltan, B. Boroomand, A. Jahed and H. Rezaie, "Performance-Based Adaptive Gradient Descent Optimal Coefficient Fuzzy Sliding Mode Methodology", International Journal of Intelligent Systems and Applications, vol. 11, (2012), pp. 40-52.

[5] B. Siciliano and O. Khatib, Springer handbook of robotics: Springer-Verlag New York Inc, (2008).

[6] F. Piltan, H. Rezaie, B. Boroomand and A. Jahed, "Design robust back stepping online tuning feedback linearization control applied to IC engine", International Journal of Advance Science and Technology, vol. 42, (2012), pp. 183-204.

[7] F. Piltan, R. Bayat, S. Mehara and J. Meigolinedjad, "GDO Artificial Intelligence-Based Switching PID Baseline Feedback Linearization Method: Controlled PUMA Workspace", International Journal of Information Engineering and Electronic Business, vol. 5, (2012), pp. 17-26.

[8] A. Vivas and V. Mosquera, "Predictive functional control of a PUMA robot", Conference Proceedings, (2005).

[9] D. Nguyen-Tuong, M. Seeger and J. Peters, "Computed torque control with nonparametric regression models", IEEE conference proceeding, (2008), pp. 212-217.

[10] B. K. Yoo and W. C. Ham, "Adaptive control of robot manipulator using fuzzy compensator", Fuzzy Systems, IEEE Transactions, vol. 8, no. 2, (2002), pp. 186-199.

[11] Y. S. Kung, C. S. Chen and G. S. Shu, "Design and Implementation of a Servo System for Robotic Manipulator", CACS, (2005).

[12] F. Piltan, M. H. Yarmahmoudi, M. Shamsodini, E. Mazlomian and A. Hosainpour, "PUMA-560 Robot Manipulator Position Computed Torque Control Methods Using MATLAB/SIMULINK and Their Integration into Graduate Nonlinear Control and MATLAB Courses", International Journal of Robotics and Automation, vol. 3, no. 3, (2012).

[13] F. Piltan, S. Emamzadeh, Z. Hivand, F. Shahriyari and M. Mirazaei, "PUMA-560 Robot Manipulator Position Sliding Mode Control Methods Using MATLAB/SIMULINK and Their Integration into Graduate/Undergraduate Nonlinear Control, Robotics and MATLAB Courses", International Journal of Robotics and Automation, vol. 3, no. 3, (2012).

[14] A. Barzegar, F. Piltan, M. Vosoogh, A. Majid Mirshekaran and A. Siahbazi, "Design Serial Intelligent Modified Feedback Linearization like Controller with Application to Spherical Motor", Journal of Information Technology and Computer Science, DOI: 10.5815/ijitcs. 2014.05.10 (DOAJ, DOI: 10.5815), vol. 6, no. 5, (2014), pp. 72-83. 
[15] F. Matin, F. Piltan, H. Cheraghi, N. Sobhani and M. Rahmani, "Design Intelligent PID like Fuzzy Sliding Mode Controller for Spherical Motor", International Journal of Information Engineering and Electronic Business, DOI: 10.5815/ijieeb. 2014.02.07(DOAJ, DOI: 10.5815), vol. 6, no. 2, (2014), pp. 53-63.

[16] M. Rahmani, F. Piltan, F. Matin, H. Cheraghi and N. Sobhani, "Design Intelligent System Compensator to Computed Torque Control of Spherical Motor", International Journal of Intelligent Systems and Applications, DOI: 10.5815/ijisa.2014.08.10 (DOAJ, DOI: 10.5815, vol. 6, no. 8, (2014), pp. 87-96.

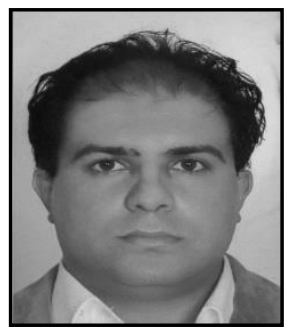

Farzin Piltan was born on 1975, Shiraz, Iran. In 2004 he is jointed Institute of Advance Science and Technology, Research and Development Center, IRAN SSP. Now he is a dean of Intelligent Control and Robotics Lab. He is led of team (47 researchers) to design and build of nonlinear control of industrial robot manipulator for experimental research and education and published about 54 Papers in this field since 2010 to 2012, team supervisor and leader (9 researchers) to design and implement intelligent tuning the rate of fuel ratio in internal combustion engine for experimental research and education and published about 17 Journal papers since 2011 to 2013, team leader and advisor (34 researchers) of filtering the hand tremors in flexible surgical robot for experimental research and education and published about 31 journal papers in this field since 2012 to date, led of team (21 researchers) to design high precision and fast dynamic controller for multi-degrees of freedom actuator for experimental research and education and published about 7 journal papers in this field since 2013 to date, led of team ( 22 researchers) to research of full digital control for nonlinear systems (e.g., Industrial Robot Manipulator, IC Engine, Continuum Robot, and Spherical Motor) for experimental research and education and published about 4 journal papers in this field since 2010 to date and finally led of team (more than 130 researchers) to implementation of Project Based-Learning project at IRAN SSP research center for experimental research and education, and published more than 110 journal papers since 2010 to date. In addition to 7 textbooks, Farzin Piltan is the main author of more than 115 scientific papers in refereed journals. He is editorial review board member for 'international journal of control and automation (IJCA), Australia, ISSN: 20054297; 'International Journal of Intelligent System and Applications (IJISA)', Hong Kong, ISSN:2074-9058; 'IAES international journal of robotics and automation, Malaysia, ISSN:2089-4856; 'International Journal of Reconfigurable and Embedded Systems', Malaysia, ISSN:2089-4864. His current research interests are nonlinear control, artificial control system and applied to FPGA, robotics and artificial nonlinear control and IC engine modeling and control.

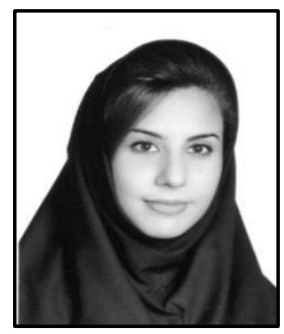

Zahra Hivand is currently a co researcher in the Intelligent System and Robotic Lab at Iranian Institute of Advance Science and Technology (IRAN SSP) of the program for "Design and build of intelligent controller for industrial robot manipulator". 


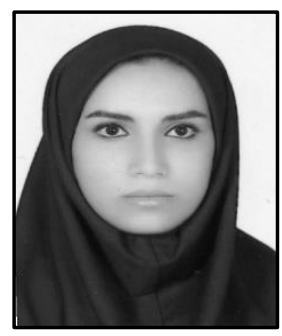

Sara Emamzadeh is currently a co researcher in the Intelligent System and Robotic Lab at Iranian Institute of Advance Science and Technology (IRAN SSP) of the program for "Design and build of intelligent controller for industrial robot manipulator".

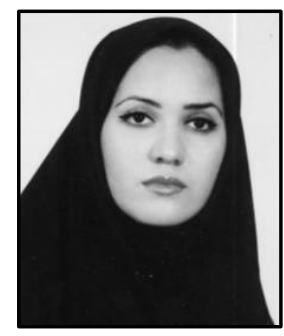

Mina Mirzaie is currently a co researcher in the Intelligent System and Robotic Lab at Iranian Institute of Advance Science and Technology (IRAN SSP) of the program for "Design and build of intelligent controller for industrial robot manipulator".

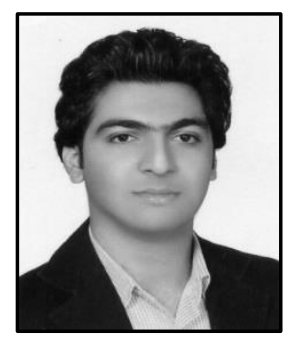

Mohammad Hossain Yarmahmoudi is currently a co researcher in the Intelligent System and Robotic Lab at Iranian Institute of Advance Science and Technology (IRAN SSP) of the program for "Design and build of intelligent controller for industrial robot manipulator". 